\title{
The Use of the Tape Recorder in an ESL Composition Programme
}

James Patrie

Although tape recorders are commonly used in ESL programmes, they are not often seen as appropriate to the goals of the composition classroom. I would like to advocate their use as an appropriate medium for feedback as part of the writing process.

Teaching composition is a time-consuming task. I have often heard ESL teachers comment that one composition course is easily equivalent to two conversation courses in terms of the out-of-class time required. Our composition students are required to write and we are required to give appropriate feedback on their writing. Many ESL programmes do not have structured into the teachers' workload student/teacher writing conferences where one-to-one contact is built into the programme of instruction. Thus responding to student compositions, either final product or intermediate draft, is restricted to the teacher's written comments and corrections on the students submission.

Particularly if we are grading a final draft, our detailed comments and corrections often seem to be unappreciated as the returned paper is quickly scanned for the assigned grade with the comments receiving only a cursory glance or even going unread. As more and more composition courses now emphasize the writing process where the teacher guides the student through a series of drafts, this is becoming less of an issue, but the point remains that successful ESL composition teaching requires a great deal of teacher input, feedback and interaction. Presenting this feedback as written comments on a student's paper is not only time-consuming but also is less personal and more distant than direct contact. Conversely, an oral conference with the writing instructor not only may unduly raise the student's affective filter, but the student, unless assiduous and skilful in taking detailed notes during the conference, will have no permanent record of the input and suggested modifications. Thus when he or she sits down to write the next draft, often hours or even days later, much of the commentary may have been forgotten.

A technique which has proven successful for me in overcoming some of the problems inherent to written feedback, is to tape record my responses. When I switched to this mode, one of the most apparent things was that I was now giving radically more feedback. Very few writing 
teachers would agree to spending only 15 minutes per writing assignment. This would be viewed as painfully inadequate to giving the quantity and quality of input necessary for the student. This is why we pity the poor teacher who goes home with 25 writing assignments that must be graded/ responded to before the next day's class. If that teacher is to successfully do the task, I think we can be assured that there will be very little sleep in that house that night. The simple fact is that it takes time to write. Another simple fact is that we talk a lot faster than we write. The implication of these observations to the issue of composition feedback can be demonstrated in two ways. First, time yourself when grading/responding to a student's composition in the traditional manner of written feedback. Now time yourself as you read your written comments out loud. What took you at least half an hour to write was probably spoken in about 5 minutes. Now do the converse. Tape your oral responses to a student's composition and now transcribe this oral passage into written form. You will find that you have given literally pages more commentary than what you ordinarily give in the written mode. You will also find that you have taken just a fraction of the time to give this substantially greater quantity of input.

Not only is there a difference in the quantity of input, but I have found that the tape recorder mode affects a substantial change in the quality and nature of the feedback. Traditional written feedback has a tendency to focus on sentence or even clause-level units of writing, and also with a focus on local, surface-level structural errors. With my red pen in hand, it is very easy to correct spelling, punctuation, missing articles, incorrect conjugation etc., and this ease often translates into a tendency for teachers to focus on this type of feedback. But note that just the opposite is the case with oral feedback. Just as it is easy, even natural, for me to mark in a missing $-s$, it is difficult and unnatural for me to orally mention that the main verb in the second sentence of your third paragraph should have the third person singular inflection. The type of feedback which is natural now becomes an examination of the content of the composition: what was actually said, how it was said, and how it was developed, as well as your reaction to the content. You will naturally respond to the writing as a whole unit of discourse and your responses will also become a dialogue between you and the writer.

I have found that students respond very favourably to this technique. The semester that I first experimented with this mode of feedback, I had students who gave a cursory glance at the final grade and then all but ignore my copious written comments, change to those who would head off to the language lab immediately after class to listen to my comments. Note, by the way, that it is unfair if you are doing a final draft evaluation to trick or force your students to listen to your commentary by not mention- 
ing their grade until your final line of commentary. It is also unnecessary. Even when you write the assigned grade on the paper, there is still a natural desire to hear what you have said about that paper. Students respond well to the oral input and treat it as a very personal response to their writing. They want to hear it and they relate well to it. Students view oral feedback as more personal whereas written feedback tends to be viewed as evaluative. I have had far more students approach me with questions and responses to my oral feedback than I have had with traditional written feedback. In addition, students still have a permanent record of your input that they can refer back to when they are doing their rewriting.

One note of caution: do not assume that your students have access to a tape recorder in their homes. ESL students who are struggling with the financial burden of resettlement may not have the money to spare for the purchase of a tape recorder. Hence ensure that appropriate resources are available at your school before you switch to this feedback technique.

\section{THE AUTHOR}

James Patrie is Associate Professor of TESL at the University of Alberta. 